\title{
A Model-Based Dead-Band Compensation for the Dual-Active-Bridge Isolated Bidirectional DC-DC Converter
}

\author{
Hua $\mathrm{Bai}^{* a}$, Non-memeber \\ Ziling Nie**, Non-member \\ Chris Chunting $\mathrm{Mi}^{* * *}$, Non-member
}

\begin{abstract}
The dual active bridge (DAB)-based isolated bidirectional converter has been used to realize bidirectional energy flow while offering needed isolation between the primary and secondary side: for example, the battery side and grid side of one plugin hybrid electric vehicle (PHEV). Even though the operation of a DAB-based DC-DC converter is straightforward, various transient processes exist, such as the dead-band effect, which deeply affects the dynamic performance of the converter in real world applications. Compensation of this effect is not easy because of the strong nonlinearity of the entire system. This paper quantitatively analyzed the dead-band effect at different output powers, and presented a model-based controller to realize the nonlinear dead-band compensation strategy, which can effectively mitigate demerits of the traditional PI-based control strategy. The proposed control algorithm is validated through theoretical simulation and experimental results. (C) 2011 Institute of Electrical Engineers of Japan. Published by John Wiley \& Sons, Inc.
\end{abstract}

Keywords: dead-band, DC-DC converter, phase-shift, PI controller, short timescale

Received 16 July 2009; Revised 14 January 2010

\section{Introduction}

In the contemporary world, energy and environmental sustainability have become a major concern. Plug-in hybrid electric vehicles (PHEVs) can address these energy and environmental concerns by using an electric grid to drive the vehicle in short distances, thereby offering better fuel economy and lower emissions than conventional vehicles. The electric propulsion power of a PHEV can originate from multiple energy sources, such as solar, wind, and nuclear. A PHEV involves multiple energy sources and converters, such as battery, ultracapacitor, electric motor, and internal combustion engine. The vehicle's fuel efficiency and performance can be effectively managed and optimized through the use of power electronic converters [1-5].

Among all power electronic systems associated with a PHEV, the DC-DC converter is a key component in power flow management. Particularly, the full-bridge converter exhibits many advantages, such as higher power and soft-switching capability. The dual active bridge (DAB)-based DC-DC converter can realize bidirectional energy flow while offering the needed isolation between the electric grid and the onboard energy storage device. The bidirectional capability of the converter enables the vehicle to be used as back-up power during a power outage, or as a peak shaving device [6-12].

Figure 1 shows the typical scheme of a DAB-based isolated bidirectional DC-DC converter. State-of-the-art research in DC-DC converters primarily concentrates on its steady-state operations, such as soft-switching topology and control. Among all of the control algorithms, phase-shift control is the most prevalent and

${ }^{\text {a }}$ Correspondence to: Hua Bai, ECE Office, Kettering University, 1700 3rd Ave, MI 48504, USA.

E-mail: kalvinbai9585@yahoo.com; hbai@kettering.edu

* Department of Electrical and Computer Engineering, Ketttering University, Flint, MI 48504, USA

** College of Electrical and Electronics Engineering, Hua Zhong University of Science and Technology, Hubei 430074, China

*** Department of Electrical and Computer Engineering, University of Michigan-Dearborn, Dearborn, MI 48141, USA highly developed algorithm. However, even for this fully matured control technique, there are still abundant issues to be addressed, especially those transient processes in high-voltage and high-power applications where semiconductors can not provide abundant voltage/current margin. Those microscopic influential factors, such as the dead-band effect, are generally neglected in low power converters, but will be of importance in high-voltage and high-power converters [13-16].

Dead-band is the typical issues affecting the steady-state operation of DAB-based converters. Previous literatures do not focus on the dead-band effect because of the small settings of dead-bands in low-voltage and low-power converters. However, in high voltage and high power systems where insulated gate bipolar transistor (IGBT) is preferred, the dead-band is becoming larger because of the semiconductor characteristics and power ratings [17-19].

It has been pointed out in the previous articles that within the dead-band, the whole system stays in a totally uncontrollable state, which will deteriorate the operational performance. In the closed-loop control of DC-DC converters, where mostly PI modulators are adopted, the modulated error caused by the dead-band will weaken the system performance. Therefore, the dead-band compensation is necessary.

This paper presents the quantificational analysis of the dead-band effect and its compensation. Section 2 analyzes the dead-band effect and presents its characteristics in DC-DC converters. In order to overcome the disadvantageous dead-band effect and reduce the relevant impact to the system, Section 3 proposes a model based dead-band compensation collaborating with a previously proposed phase-shift predictor. The other influential factors affecting the compensating algorithm are also detailed in subsequent sections [20].

\section{Dead-Band Effect}

In order to prevent the shoot-through during the commutating process, a dead time, $T_{\mathrm{d}}$, is inserted between the interlocked switches in Fig. 1. However, in the light-load condition, this dead-band will affect the time sequence among different IGBT's 


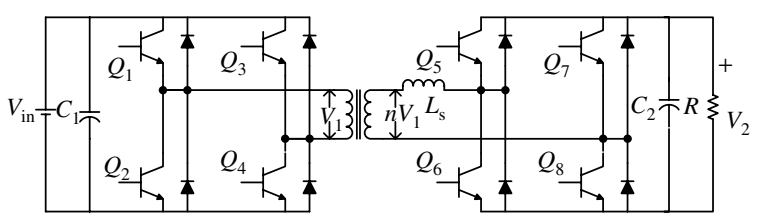

Fig. 1. The isolated bidirectional DC-DC converter in a PHEV

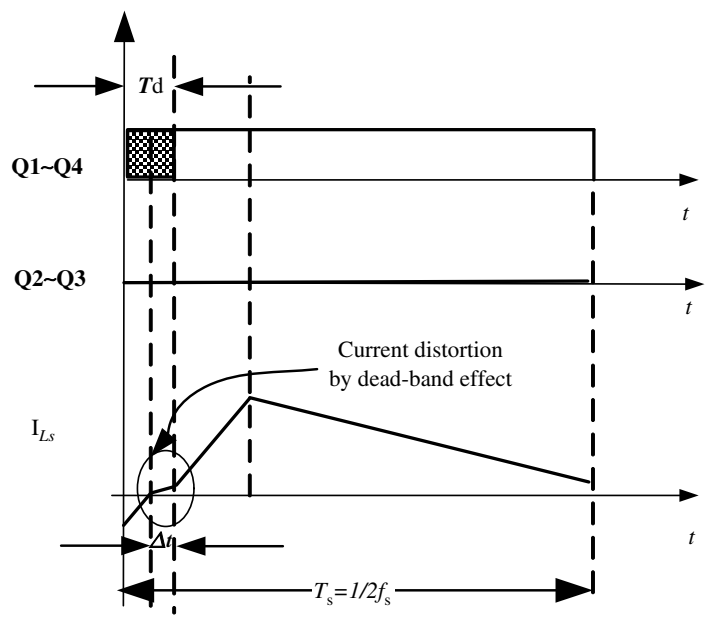

Fig. 2. Influence of the dead-band effect

gate signals. It therefore affects the operational performance. In this paper, the phase-shift ratio $D$ is modulated by a PI controller, whose input is the difference between the desired output and the actual output voltage, i.e. $V_{2}-V_{2} *$. Here, $V_{2} *$ is the referenced output voltage.

In the dead-band, the $H$ bridge behaves as a rectifier, and the polarity of the voltage imposed on the primary/secondary terminals depends on the direction of $i\left(t_{0}\right)$, as shown in Fig. 2. Before current changes from negative to positive, the current slope is $\left(n V_{1}+V_{2}\right) / L_{\mathrm{s}}$. After that, it becomes $\left(-n V_{1}+V_{2}\right) / L_{\mathrm{s}}$. Then, it changes back to $\left(n V_{1}+V_{2}\right) / L_{\mathrm{s}}$.

Compared to the dead-band effect in motor control, the DAB-based DC-DC converter has its own special characteristics. In a motor drive, the dead-band effect will occur in both light-load and heavy-load conditions. However, from the previous paper, the dead-band will erase part of the shifted phase angle between Q1 and Q5 in the light-load conditions, not in the heavy-load conditions. In addition, this erased angle is a nonlinear function of the load, which will be depicted in the following sections. Another difference of the dead-band effect between a DAB-based bidirectional converter and a motor control lies on the current direction. In motor control, the current will mostly maintain the same direction in one switching period. However, in the phase-shift-based DC-DC converter shown in Fig. 1, the current oscillates at a high frequency. Even in the dead-band, the current will change its direction, which prohibits similar dead-band compensating algorithms used in motor control, unless other advanced control algorithms are implemented to maintain the current direction in the whole dead-band [18,21,22].

The power delivered by the converter shown in Fig. 1 based on the phase-shift control is [21]

$$
P=\frac{n V_{1} V_{2}}{2 \pi^{2} f_{\mathrm{s}} L_{\mathrm{s}}} \phi(\pi-|\phi|)=\frac{n V_{1} V_{2}}{2 f_{\mathrm{s}} L_{\mathrm{s}}} D(1-|D|)
$$

$\Phi$ is the phase-shift angle. Suppose $m=V_{2} /\left(n V_{1}\right)>1$, then,

$$
i(0)=i\left(t_{0}\right)=\frac{1}{4 L_{\mathrm{s}} f_{\mathrm{s}}}\left[(1-2 D) V_{2}-n V_{1}\right]
$$

Solve $D$ in (2) and substitute it in (1), the relationship between power and $\Delta t$ can be obtained and is shown in Table I. $t_{0}$ is the initial point when Q1 and Q4 is turned on.

Table I shows that the erased time, $\Delta t$ by dead-band $T_{\mathrm{d}}$, is a nonlinear function of output power $P$. The simulations and calculations are illustrated in Fig. 3 where the phase-shift difference $\Delta t$ is exactly the same as $T_{\mathrm{d}}$ at a very light load, and zero at a heavy load. At medium output power, the simulation and calculation results show good agreement. The difference will be illustrated in Section 3.

Therefore, if the commanded power $P$ is known, then phaseshift $\Delta t$ erased by the dead-band can be derived for dead-band compensation.

\section{Dead-Band Compensation Based on a Phase-Shift Predictor}

An earlier study showed that dead-band will affect light-load operations more than heavy-load operations due to the erased phase-shift angle between the primary and secondary voltage of the isolated transformer. In the steady-state operation, the PI modulator can automatically compensate the phase-shift error caused by the dead-band. In this paper, the dead-band compensation is not aimed to compensate the angle erased by the dead-band in the steady state, but is targeted to obtain more satisfactory dynamic performances [18].

In ref. [20], we proposed a phase-shift predictor to mitigate the difficulties of the PI controller parameter selection, shown as Controllers I and II in Fig. 4. In Controller I, the output voltage and current are sampled to calculate the demanded power. Then the phase-shift is calculated by (1). In Controller II, the output voltage/current is sampled and delivered to a PI controller, which generates the phase-shift directly to the gate drive signals. Essentially, this is a feed-back and feed-forward control, whose foundation is the online identification of load resistance. This control is to preset a value $D^{*}$, close to the real phase-shift ratio by load identification. However, the identified $D^{*}$ is only a theoretical value without the consideration of dead-band $T_{\mathrm{d}}$, i.e. the phaseshift between the primary voltage and secondary voltage of the transformer. What the control algorithm handles directly is the phase-shift between Q1 and Q5, not the shift angle between the primary and secondary voltage. At light-load conditions where $D^{*}$ is close to zero, the output of the PI controller will still be dominant. For example, when $P=1 \mathrm{~kW}$, the ideal phase-shift is

Table I. Relationship between power and phase-shift by an erased dead-band

\begin{tabular}{lcc}
\hline$i(0)$ & Power $(P)$ & Erased shift time $(\Delta t)$ \\
\hline$i(0)<-\frac{T_{\mathrm{d}}}{L_{\mathrm{S}}}\left(n V_{1}+V_{2}\right)$ & $P>\frac{n V_{1} V_{2}}{8 f_{\mathrm{s}} L_{\mathrm{s}}}\left[1-\left(\frac{1-4 f_{\mathrm{s}} T_{\mathrm{d}}(1+m)}{m}\right)^{2}\right]$ & $\Delta t=0$ \\
$-\frac{T_{\mathrm{d}}}{L_{\mathrm{S}}}\left(n V_{1}+V_{2}\right) \leq i(0)<0$ & $\frac{n V_{1} V_{2}}{8 f_{\mathrm{s}} L_{\mathrm{s}}}\left[1-\left(\frac{1}{m}\right)^{2}\right]<P \leq \frac{n V_{1} V_{2}}{8 \mathrm{~s}_{\mathrm{s}}}\left[1-\left(\frac{1-4 f_{\mathrm{s}} T_{\mathrm{d}}(1+m)}{m}\right)^{2}\right]$ & $\Delta t=T_{\mathrm{d}}-\frac{1-\sqrt{1-\frac{8 f \mathrm{~s} \mathrm{~S}_{\mathrm{S}}}{n} V_{1} V_{2}} m}{4(1+m) f_{\mathrm{s}}}$ \\
$i(0) \geq 0$ & $P \leq \frac{n V_{1} V_{2}}{8 f_{\mathrm{s}} L_{\mathrm{s}}}\left[1-\left(\frac{1}{m}\right)^{2}\right]$ & $\Delta t=T_{\mathrm{d}}$
\end{tabular}




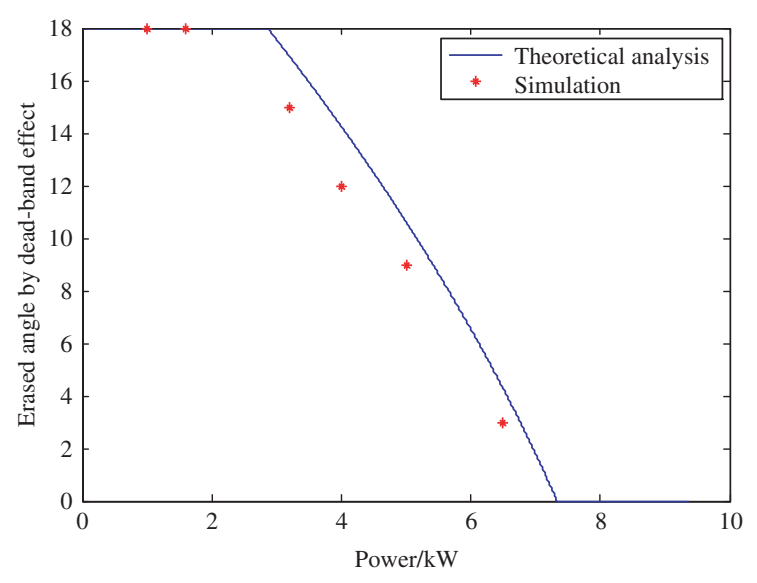

Fig. 3. Erased angle by the dead-band

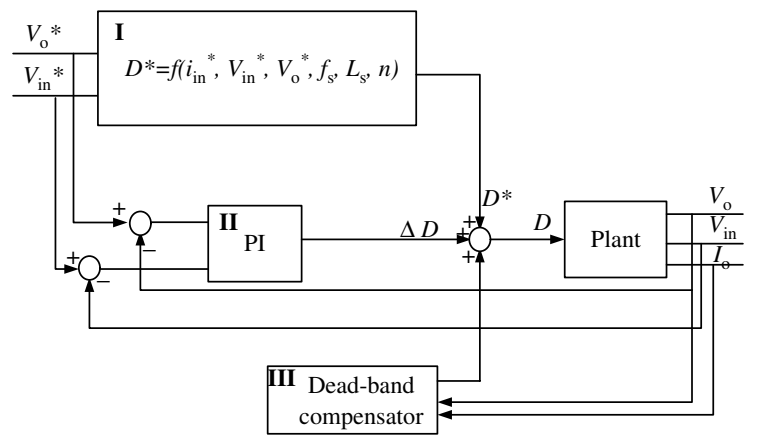

Fig. 4. Dead-band compensator based on a phase-shift predictor

$11_{\mathrm{o}}$. However, due to the existence of dead-band, the real angle between Q1 and Q5 is $29^{\circ}$ when switching frequency is $10 \mathrm{kHz}$ and the dead-band is $5 \mu \mathrm{s}$, which means that the PI controller needs to modulate approximately $18^{\circ}$ [20].

Therefore, a dead-band compensator is intended to further decrease the proportion of the PI controller, shown as Controller III in Fig. 4. In order to implement Controllers II and III in Fig. 4, load resistance should be precisely identified. Here, one current sensor and one voltage sensor are utilized to sample the load voltage $V_{\mathrm{o}}$ and current $I_{\mathrm{o}}$, respectively, thereby obtaining the load resistance online, i.e. $R=V_{\mathrm{o}} / I_{\mathrm{O}}$.

It was shown that output power determines the current direction, and current direction determines the angle erased by the dead-band effect. Therefore, besides load identification, another important issue is to precisely determine the erased angle at different levels of output power. In this process, the excitation inductance of the transformer needs to be considered, especially under light-load conditions where the excitation current is comparable to the load current. The existence of excitation current may change the current waveform, direction, and magnitude, which is of importance to dead-band compensation. Therefore, the model of the isolated transformer with mutual inductance is used to simulate the phaseshift erased by the dead-band, as shown in Fig. 5(a) [21].

Consider the transformer model in Fig. 5(a) and re-derive the relationship between $i(0)$ and $P$. Therefore, Table $\mathrm{I}$ is amended as Table II, where $V_{2}^{\prime}=\frac{L_{m}}{L_{2}+L_{m}} V_{2}, L_{\mathrm{s}}^{\prime}=L_{1}^{\prime}+\frac{L_{2} L_{m}}{L_{2}+L_{m}}$, and $m^{\prime}=$ $\frac{L_{m}}{L_{2}+L_{m}} \frac{V_{2}}{n V_{1}}>1 . L_{1}^{\prime}$ is the equivalent leakage inductance from the primary side to secondary side. $L_{2}$ is the secondary side inductance, and $L_{m}$ is the excitation inductance. The erased angles based on the isolated transformer model with/without considering the influence of excitation current are shown at different output power in Fig. 5(b). Simulation shows that Table II has better agreement with the theoretical calculations than Table I.

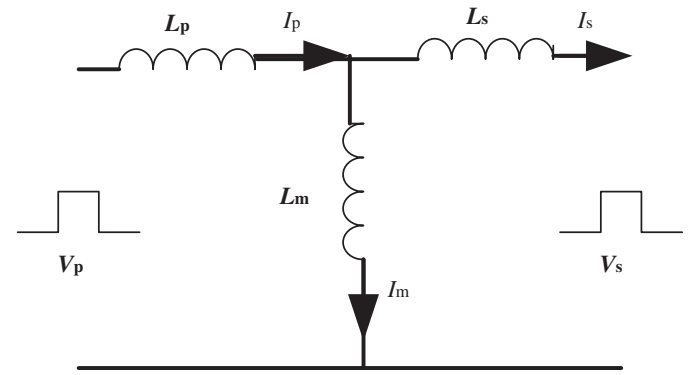

(a) Transformer model with exciting inductance

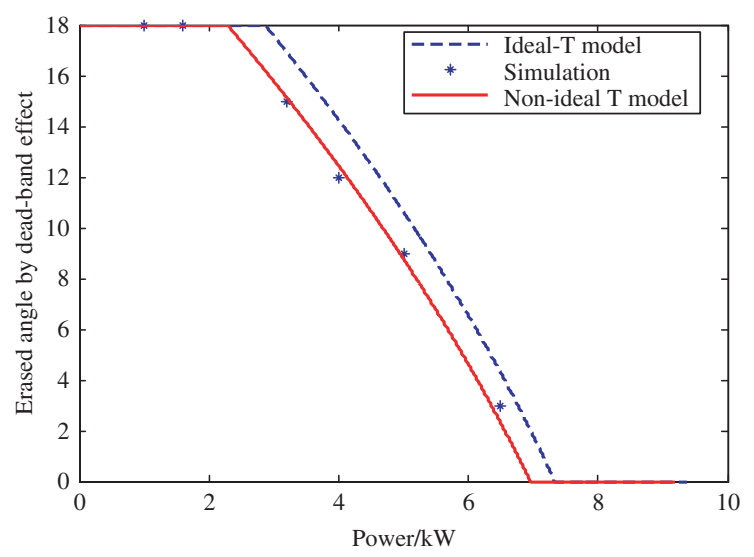

(b) Adjustment of dead-band compensation

Fig. 5. Erased angle under using different models for the isolation transformer

Here we compare three control strategies based on the same PI parameters. Control 1 (Controller I in Fig. 4), Control 2 (Controllers I + II in Fig. 4), and Control 3 (Controllers I + II + III in Fig. 4). Comparisons of these control algorithms are listed as follows, where the initial output voltage is $600 \mathrm{~V}$ and the initial power is $600 \mathrm{~W}(R=600 \Omega)$. At $t=0.02 \mathrm{~s}, R$ is changed from 600 to $150 \Omega$, i.e. $P$ is increased from $600 \mathrm{~W}$ to $2.4 \mathrm{~kW}$.

It is evident in Fig. 6(a) that with the same PI parameters traditional PI control presents the worst dynamic performance, while the proposed phase-shift predictor with dead-band compensation behaves the best. The system based on PI control has a voltage drop of $6 \mathrm{~V}$ at $t=0.02 \mathrm{~s}$ and that based on phase-shift predictor with dead-band compensation has less than $1 \mathrm{~V}$. More importantly, there is no significant current peak for the proposed control when the load is suddenly increased, yet for traditional PI control, there is a big current leap as shown in Fig. 6(b) and (d). Simulation shows that the proposed control has the best dynamic performance. In the steady state, the static error and ripples are determined by the PI controller, hence there is no big difference among these strategies in the steady-state operation. In this simulation, the time for sampling voltage, calculating phase, and implementing the control is not considered. Therefore, simulation results might show the difference from the experimental ones, as shown in the latter section.

The proposed phase-shift predictor plus dead-band compensation will not decrease the steady-state ripple of the output voltage because this is determined by the PI parameters. However, this control algorithm will improve dynamic performance. Therefore, it is possible to optimize the size of the output capacitance $C_{2}$. Meanwhile, less effort is needed in optimizing the PI parameters. For example, in Fig. 1 the output capacitance $C_{2}$ was reduced from 1800 to $600 \mu \mathrm{F}$, and the PI parameters are selected roughly without any optimization. For the traditional PI controller, when the load is instantaneously increased from 1.2 to $9 \mathrm{~kW}$, the voltage drop is significant $(12 \mathrm{~V})$. For the phase-shift predictor, the voltage drop is decreased to $6 \mathrm{~V}$. For the dead-band compensation based on 
Table II. Relationship between power and time interval to compensate (revised)

\begin{tabular}{lcc}
\hline$i(0)$ & Power $(P)$ & Erased shift time $(\Delta t)$ \\
\hline$i(0)<-\frac{T_{\mathrm{d}}}{L_{2}^{\prime}}\left(n V_{1}+V_{2}^{\prime}\right)$ & $P>\frac{n V_{1} V_{2}^{\prime}}{8 f_{\mathrm{s}} L_{\mathrm{S}}^{\prime}}\left[1-\left(\frac{1-4 f_{\mathrm{s}} T_{\mathrm{d}}\left(1+m^{\prime}\right)}{m^{\prime}}\right)^{2}\right]$ & $\Delta t=0$ \\
$-\frac{T_{\mathrm{d}}}{L_{S}^{\prime}}\left(n V_{1}+V_{2}^{\prime}\right) \leq i(0)<0$ & $\frac{n V_{1} V_{2}^{\prime}}{8 f_{\mathrm{s}} L_{\mathrm{S}}^{\prime}}\left[1-\left(\frac{1}{m^{\prime}}\right)^{2}\right]<P \leq \frac{n V_{1} V_{2}^{\prime}}{8 f_{s} L_{\mathrm{S}}^{\prime}}\left[1-\left(\frac{1-4 f_{\mathrm{s}} T_{\mathrm{d}}\left(1+m^{\prime}\right)}{m^{\prime}}\right)^{2}\right]$ & $\Delta t=T_{\mathrm{d}}-\frac{\sqrt{1-\frac{8 f_{\mathrm{s}} L_{\mathrm{S}}^{\prime} P}{n V_{\mathrm{s}} V_{2}^{\prime}}}}{4\left(1+m^{\prime}\right) f_{\mathrm{s}}}$ \\
$i(0) \geq 0$ & $P \leq \frac{n V_{1} V_{2}^{\prime}}{8 f_{s} L_{\mathrm{S}}^{\prime}}\left[1-\left(\frac{1}{m^{\prime}}\right)^{2}\right]$ & $\Delta t=T_{\mathrm{d}}$ \\
\hline
\end{tabular}

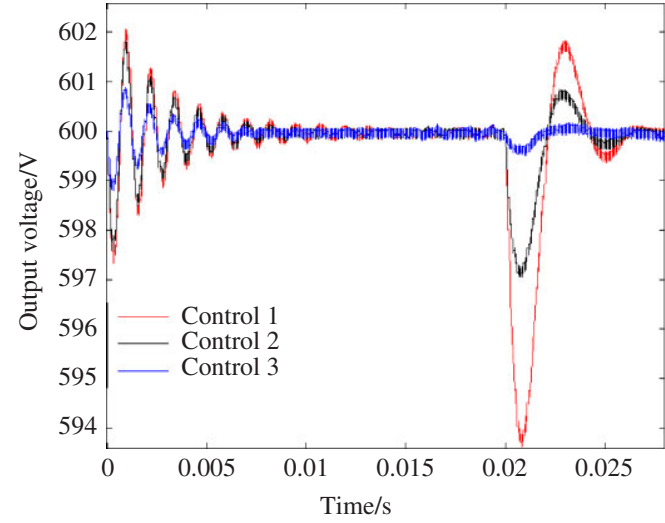

(a) Output voltage $V_{2}$

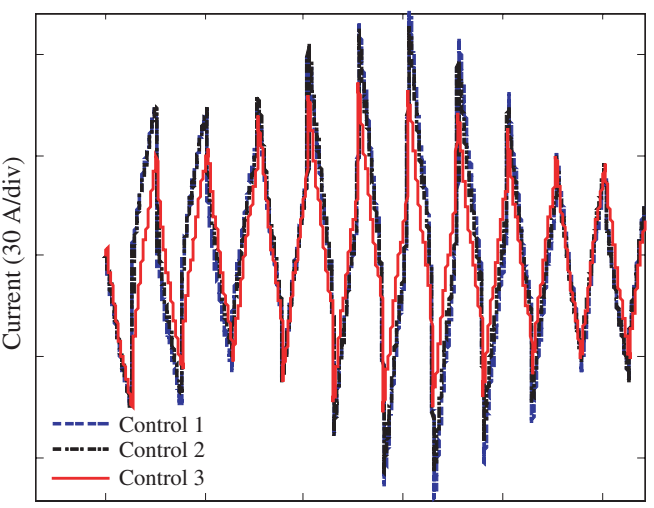

Time $(200 \mu \mathrm{s} / \mathrm{div})$

(c) Current oscillation at $t=0$

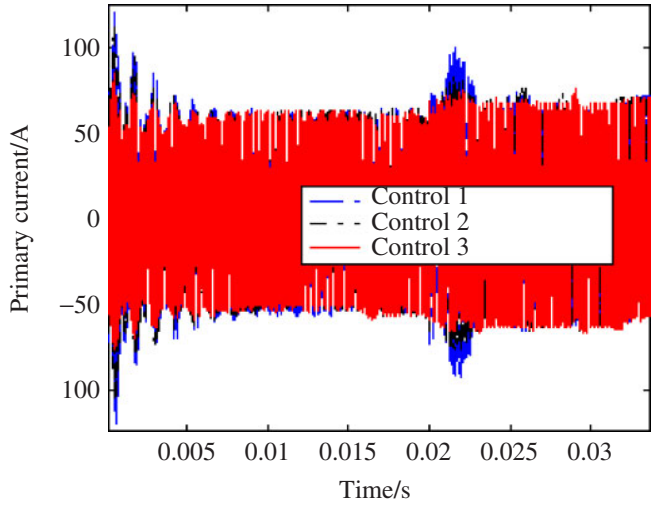

(b) Primary current

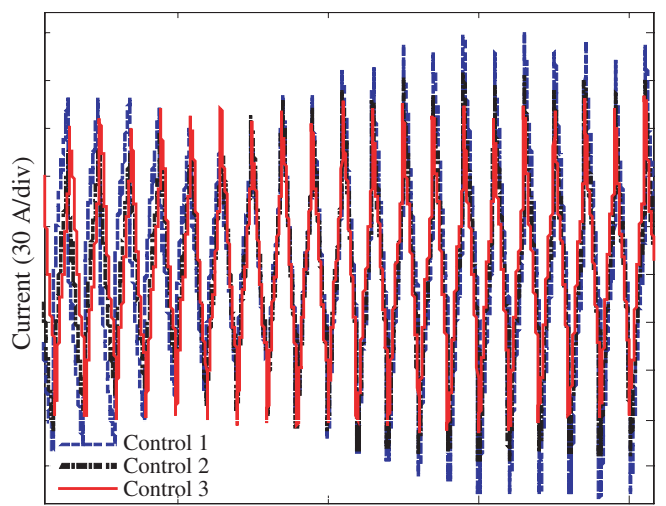

Time $(500 \mu \mathrm{s} / \mathrm{div})$

(d) Current oscillation at $t=0.02 \mathrm{~s}$

Fig. 6. Comparison of different control algorithms $\left(C_{2}=1800 \mu \mathrm{F}\right)$

the predictor, the voltage drop is nearly negligible. In Fig. 7(b), the output values of the three different controllers in Fig. 4 are compared. It can be seen that the phase shift modulated by the PI modulator is close to zero, where 1 represents phase-shift of $180^{\circ}$. Therefore, the other two modulators, i.e. the phase-shift predictor and dead-band compensator behave dominantly.

To further investigate the dynamic performance of different control strategies, a simulation comparison is given where at $t=0.02 \mathrm{~s}$ $P$ is increased from 1.2 to $9 \mathrm{~kW}$ and at $t=0.04 \mathrm{~s}, P$ is decreased from 9 to $1.2 \mathrm{~kW}$, as shown in Fig. 9 , where $C_{2}=1800 \mu \mathrm{F}$. From Fig. 8(c) and (d), it can be seen that the current modulated by the proposed algorithm reaches steady state within very short modulating process compared to the traditional PI controller.

In Fig. 6(b), the power is changed from $600 \mathrm{~W}$ to $2.4 \mathrm{~kW}$, and the current peak in the load-switched moment is small. However, it is worthwhile to point out that in Fig. 8(c), there is a big current peak when load is switched from 1.2 to $9 \mathrm{~kW}$ regardless of the short modulating process. Enhancing the dynamic response will result in a big current peak, which is not dominant in the low power operations but will impact the system in the high-power applications.

\section{Stability Analysis}

Another key issue is the small-signal model to process the stability analysis. Although the total phase-shift control is comprised of three independent controllers instead of one pure PI controller, the output of two controllers are maintained constant in the steady state, i.e. the output of phase-shift predictor and dead-band compensator. Therefore, the stability of the whole control strategy is still determined by the PI controller [20].

Simplify Fig. 1 as follows. Here, $R_{\mathrm{S}}$ is the source internal resistance.

As pointed out in ref. [20], the small-signal model of Fig. 9 is

$$
\begin{aligned}
{\left[\begin{array}{c}
\frac{\mathrm{d}\left\langle v_{1}\right\rangle}{\mathrm{d} t} \\
\frac{\mathrm{d}\left\langle v_{2}\right\rangle}{\mathrm{d} t}
\end{array}\right]=} & {\left[\begin{array}{cc}
-\frac{1}{r_{\mathrm{s}} C_{1}} & \frac{\left(D^{2}-D\right)}{2 L f_{\mathrm{s}} C_{1}} \\
\frac{\left(-D^{2}+D\right)}{2 L f_{\mathrm{s}} C_{2}} & -\frac{1}{\mathrm{RC}}
\end{array}\right]\left[\begin{array}{c}
\left\langle v_{1}\right\rangle \\
\left\langle v_{2}\right\rangle
\end{array}\right] } \\
& +\left[\begin{array}{cc}
\frac{1}{r_{\mathrm{s}} C_{1}} & \frac{2 D-1}{2 L f_{\mathrm{s}} C_{1}}\left\langle v_{2}\right\rangle \\
0 & \frac{-2 D+1}{2 L f_{\mathrm{s}} C_{2}}\left\langle v_{1}\right\rangle
\end{array}\right]\left[\begin{array}{c}
\left\langle v_{\mathrm{s}}\right\rangle \\
\langle\Delta * D\rangle
\end{array}\right]
\end{aligned}
$$

Here $\left\langle V_{1}\right\rangle$ and $\left\langle V_{2}\right\rangle$ are the averaged voltage on the primary capacitor and secondary capacitor, respectively. $D$ is the 


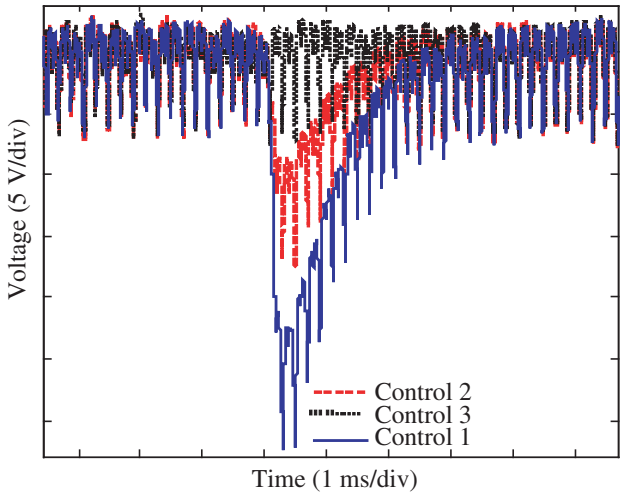

(a) Voltage drop when $P=1.2 \mathrm{~kW} \rightarrow 9 \mathrm{~kW}$

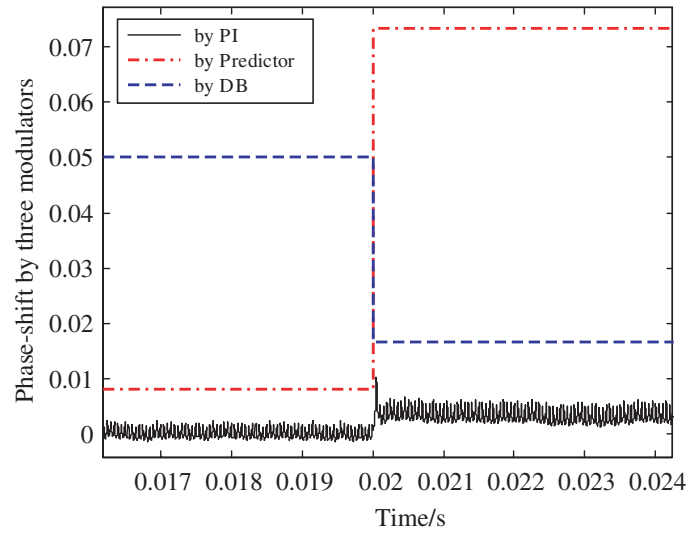

(b) Phase-shift by three modulators

Fig. 7. Comparison of different control algorithms $\left(C_{2}=600 \mu \mathrm{F}\right)$

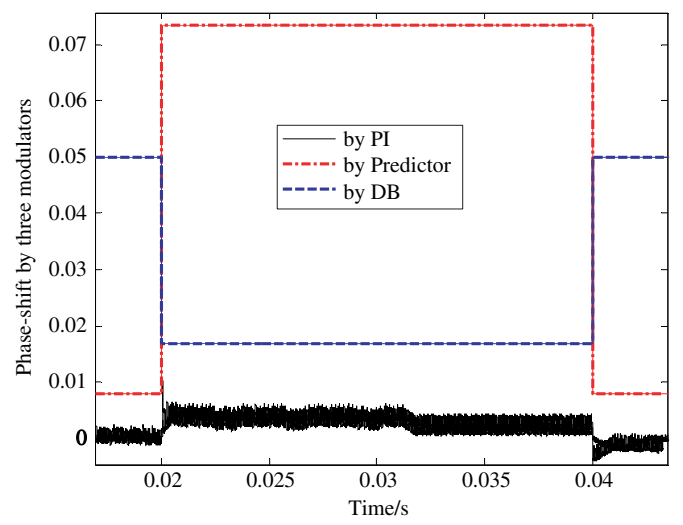

(a) Phase-shift by three modulators

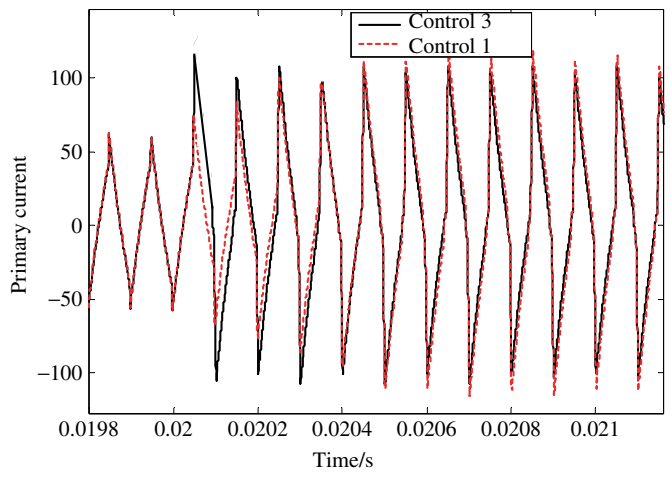

(c) Current oscillation at $t=0.02 \mathrm{~s}, P=1.2 \mathrm{~kW} \rightarrow 9 \mathrm{~kW}$

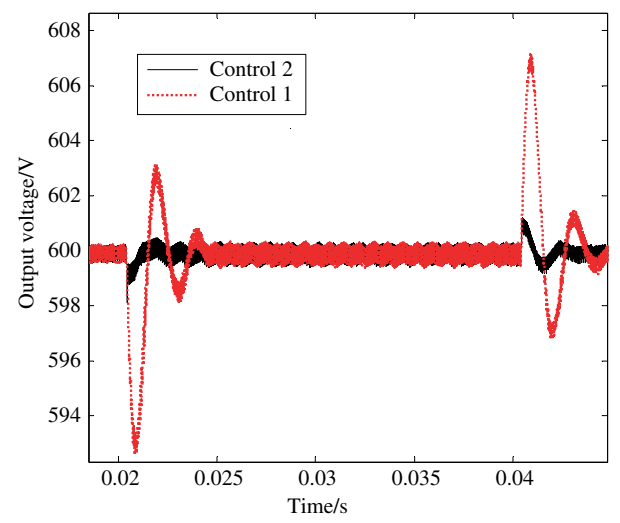

(b) Voltage variations in the dynamic process

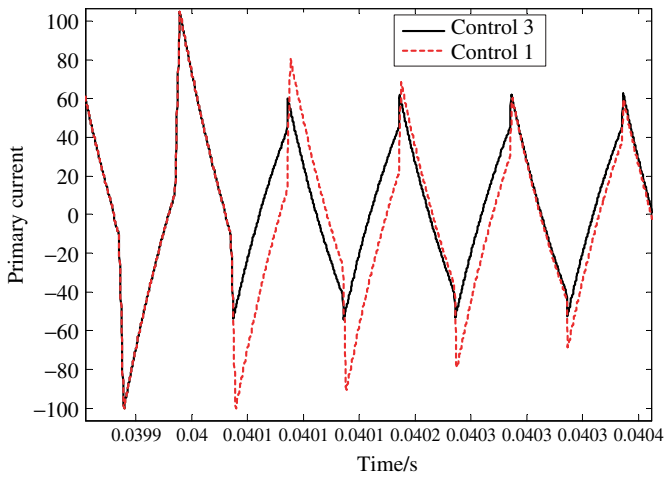

(d) Current oscillation at $t=0.04 \mathrm{~s}, P=9 \mathrm{~kW} \rightarrow 1.2 \mathrm{~kW}$

Fig. 8. Dynamic response based on the phase-shift predictor plus dead-band compensator

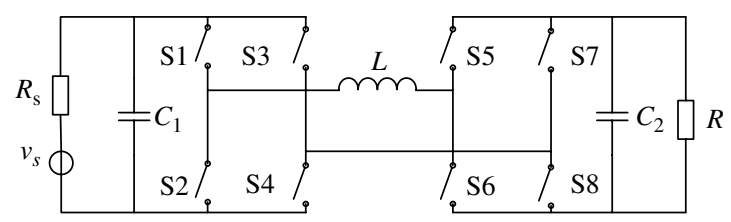

Fig. 9. Simplified circuit of a DAB DC-DC converter

output of PI controller and $\Delta$ is the relative percentage of phaseshift variation. This output voltage ripple is directly affected by the variation of phase-shift duty ratio.

$$
\frac{\Delta v_{2}}{\left\langle v_{2}\right\rangle}=\frac{-2 D+1}{4 L f_{\mathrm{s}}^{2} C_{2}} \times \frac{\left\langle v_{1}\right\rangle}{\left\langle v_{2}\right\rangle} \Delta \times D
$$

However, if the $D$ is the composition of three controllers, i.e. the dead-band compensator, the phase-shift predictor and PI controller,
$D=D_{\mathrm{PS}}+D_{\mathrm{DB}}+D_{\mathrm{PI}}$. Here, $D_{\mathrm{PS}}$ is the output of phase-shift predictor, $D_{\mathrm{DB}}$ is the output of dead-band compensator, and $D_{\mathrm{PI}}$ is the output of the PI controller. In the steady state, the $D_{\mathrm{PS}}$ and $D_{\mathrm{DB}}$ can be regarded as the constant. The only variation happens on the PI controller output. Therefore, we rewrite (4) as

$$
\frac{\Delta v_{2}}{\left\langle v_{2}\right\rangle}=\frac{-2 D+1}{4 L f_{\mathrm{s}}^{2} C_{2}} \times \frac{\left\langle v_{1}\right\rangle}{\left\langle v_{2}\right\rangle} \Delta \times D_{P I}
$$

Assume the relative variation of $D_{\mathrm{PI}}$ is the same. Comparing (5) to (4), since the $D_{\mathrm{PI}}$ is far less than $D_{\mathrm{PS}}$ and $D_{\mathrm{DB}}$, the variation of output voltage is smaller than that generated by the traditional PI-based control. Therefore, the stability of this newly proposed control is better than traditional one.

For example, the real phase shift is $D=0.3$. If this phase shift is modulated by a single PI controller, the overshoot is $20 \%$, then the maximum phase shift is 0.36 . However, with the control 3, 
the predicted phase shift is 0.2 , and the dead-band compensation is 0.05 ; therefore, the PI controller only contributes 0.05 . In the transient process, the maximum phase shift is $0.2+0.05+0.05 \times$ $(1+20 \%)=0.31$. Therefore, the overshoot of the phase shift is much smaller than one single PI controller.

\section{Experimental Results}

Due to the limitation of the experimental conditions, preliminary experiments are carried out under low voltage and low power for theoretical test. System parameters are listed as the following: $f_{\mathrm{s}}=$ $10 \mathrm{kHz}, L_{\mathrm{s}}=54 \mu \mathrm{H}, V_{\text {in }}=30 \mathrm{~V}, V_{\mathrm{o}}=70 \mathrm{~V}, n=2$. At some moments, the output capacitor of the system is switched to 61 $\Omega$. Experimental results are shown in Fig. 10.

In Fig. 10, the PI parameters are selected randomly. When the load is switched on, the control strategy of phase-shift predictor plus dead-band compensation shows the best dynamic performance, i.e. the smallest voltage drop and fastest modulating speed. The PI control behaves the worst: the biggest voltage drop and longest modulating process. In Fig. 10(b), the pure PI control behaves better than Fig. 10(a), however it still can not compete with the phase-shift predictor plus dead-band compensation. Therefore, this proposed strategy saves the effort of optimizing the PI parameters.

When power increases, e.g. the load resistance of the system is switched from open-circuit condition to $30 \Omega$, the experimental results are shown as the follows.

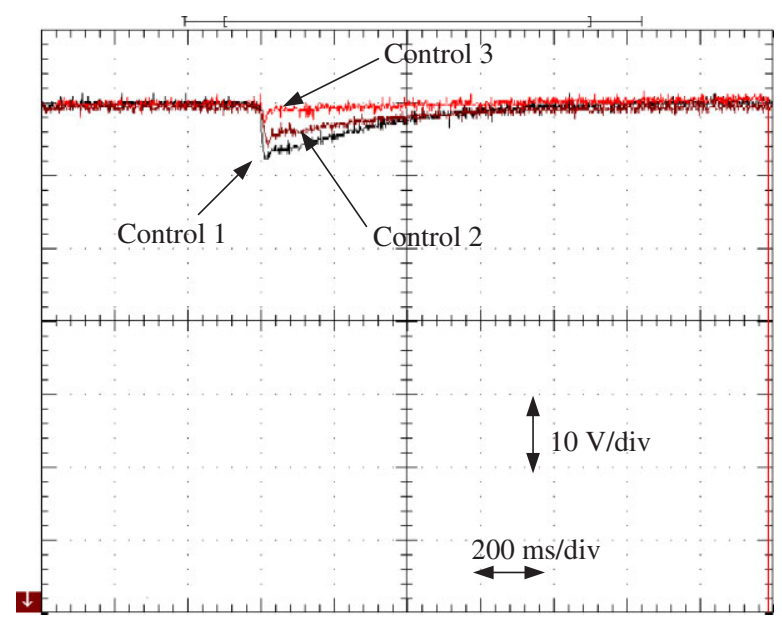

(a) $K_{p}=0.01, K_{i}=0.01$

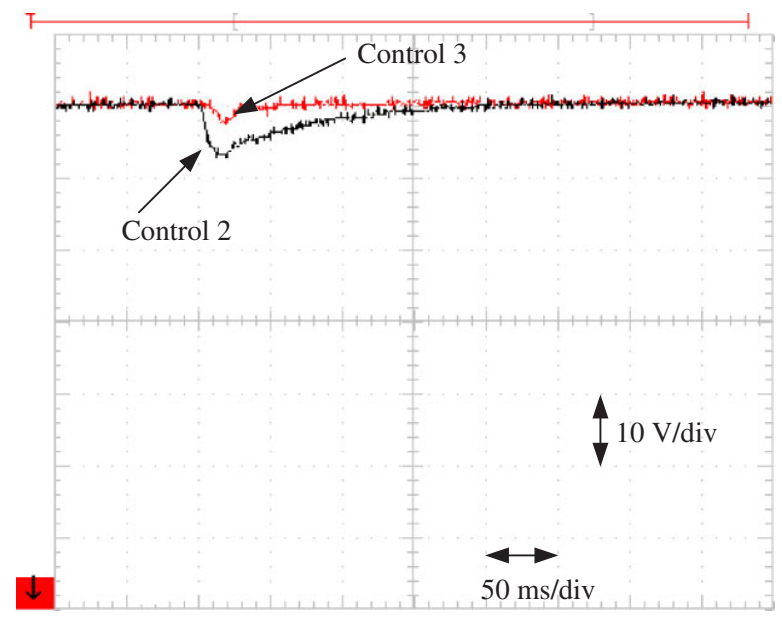

(b) $K_{p}=0.05, K_{i}=0.002$

Fig. 10. Comparisons of the control algorithms with different PI parameters

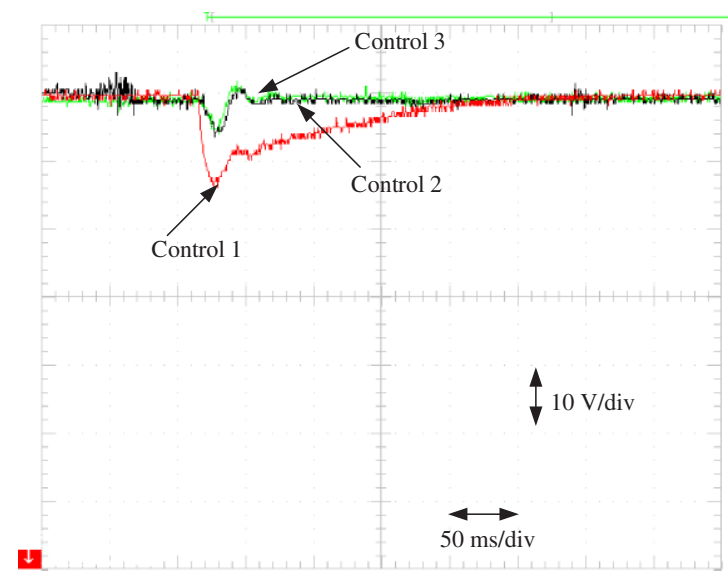

Fig. 11. Comparisons of the control algorithms when switched resistance is $30 \Omega$

As shown in Fig. 11, when the switched load increases, the voltage drops based on the three control algorithms are all increased. Single PI control still behaves the worst performance. For the other two control algorithms, i.e. the phase-shift predictor with/without dead-band compensation, their performance is nearly the same. This is because under the high load, dead-band effect disappears, thus the dead-band compensation is not needed any more.

Although the selection of the PI parameters in this algorithm, model-based dead-band compensation is not quite fatal to the dynamic performance of the whole system as single PI controller, it is still necessary to investigate the influence of the PI parameters. The simulated and experimental voltage drops when the device changes from no-load operation to $61 \Omega$ load resistance are shown as the follows. Output voltage is $70 \mathrm{~V}$. In order to reach the coincidence, the PI parameters here are the same with those adopted in Fig. 10(a) and (b).

Control 1 is the single PI control. Control 2 is the PI + phase-shift predictor. Control 3 is the dead-band compensation + phase-shift predictor + PI control. The PI parameters in Fig. 12(a) and (b) are the same with those in Fig. 10(a) and (b), respectively. In order to improve the dynamic response, as shown in Fig. 12(b), the $K_{\mathrm{p}}$, proportional coefficient is increased while the $K_{\mathrm{i}}$, integral coefficient remains the same. Therefore, the voltage drops of these three control algorithms are all improved. However, whatever the PI parameters change, Control 3 behaves more excellent than other two algorithms both in simulation and in experiments.

It is worthwhile to point out that the simulated results are superior to the experimental results. Part of the reasons is the voltage drop on the semiconductor switches, the leakage resistance of the transformer, and the parasitic parameters of the output capacitor are not included. Those factors are more dominant at the low-voltage operation. On the other hand, sampling voltage, calculating the phase-shift, and implementing the control algorithms in the real applications need the time therefore bring the time delay from voltage drop to the real PI modulation, which also contributes to the difference between experiments and simulations.

\section{Conclusions}

On the basis of the previous work of short-timescale transient processes in the full bridge DC-DC converter, this paper studied the dead-band effect quantitatively. It also proposed a control strategy to compensate the phase-shift difference caused by the dead-band, thereby avoiding disadvantages of the traditional PI 


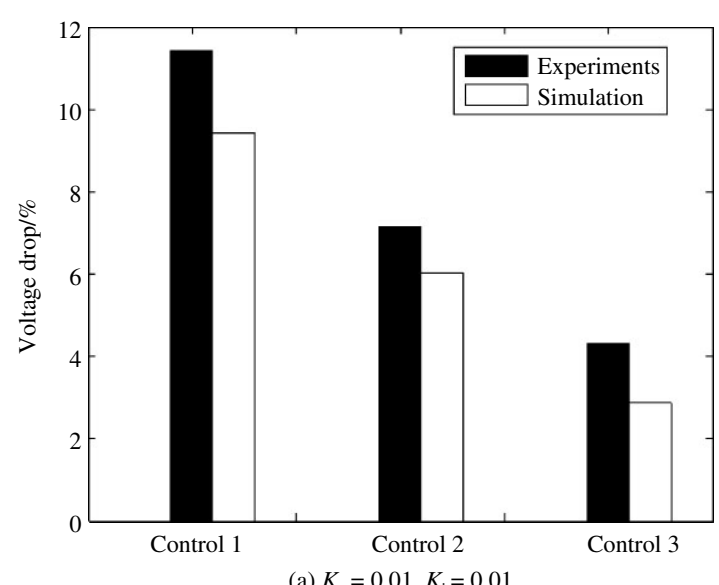

(a) $K_{\mathrm{p}}=0.01, K_{\mathrm{i}}=0.01$

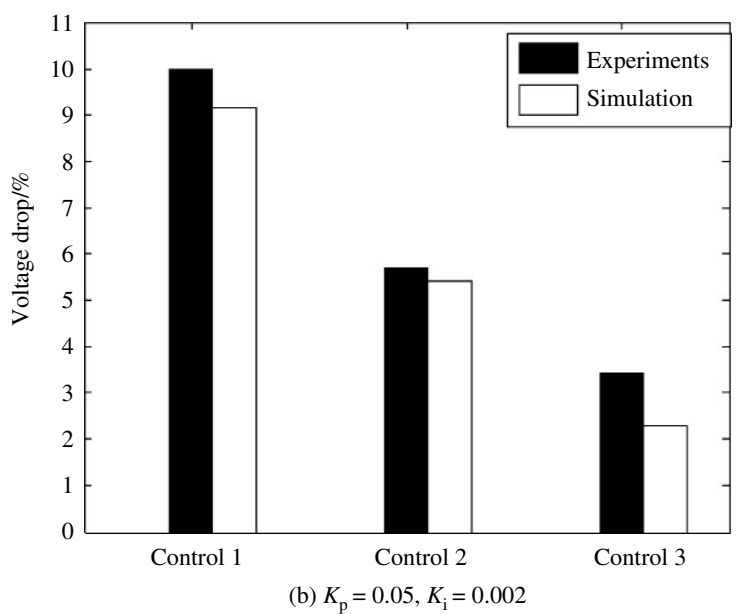

Fig. 12. Simulation and experimental results with different PI parameters

controller and enhancing the preciously proposed phase-shift predictor. With the proposed control algorithm, the dead-band effect can be compensated precisely and the dynamic response of the system can be significantly improved. Preliminary experiments showed good coincidence with the theoretical analysis and simulation. Further work needs to be expanded in the high-voltage and high-power applications.

\section{References}

(1) Emadi A, Lee YJ, Rajashekara K. Power electronics and motor drives in electric, hybrid electric, and plug-in hybrid electric vehicles. IEEE Transactions on Industrial Electronics 2008; 55(6):2237-2245.

(2) Gong Q, Li Y, Peng Z-R. Trip-based optimal power management of plug-in hybrid electric vehicles. IEEE Transactions on Vehicular Technology 2008; 57(6):3393-3401.

(3) Bhattacharya T, Giri VS, Mathew K, Umanand L. Multiphase bidirectional flyback converter topology for hybrid electric vehicles. IEEE Transactions on Industrial Electronics 2009; 56(1):78-84.

(4) Moreno J, Ortuzar ME, Dixon JW. Energy-management system for a hybrid electric vehicle, using ultracapacitors and neural networks. IEEE Transactions on Industrial Electronics 2006; 53(2):614-623.

(5) Chau KT, Chan CC, Chunhua L. Overview of permanent-magnet brushless drives for electric and hybrid electric vehicles. IEEE Transactions on Industrial Electronics 2008; 55(6):2246-2257.

(6) Yoon H-K, Han S-K, Choi E-S, Moon G-W, Youn M-J. Zero-voltage switching and soft-commutating two-transformer full-bridge PWM converter using the voltage-ripple. IEEE Transactions on Industrial Electronics 2008; 55(3):1478-1488.
(7) Kim SY, Nam K, Song H-S, Kim H-G. Fault diagnosis of a ZVS DC-DC converter based on DC-link current pulse shapes. IEEE Transactions on Industrial Electronics 2008; 55(3):1491-1494.

(8) Park K-B, Kim C-E, Moon G-W, Youn M-J. Voltage oscillation reduction technique for phase-shift full-bridge converter. IEEE Transactions on Industrial Electronics 2007; 54(5):2779-2790.

(9) Lee J-P, Min B-D, Tae-Jin K, Dong-Wook Y, Ji-Yoon Y. A novel topology for photovoltaic DC/DC full-bridge converter with flat efficiency under wide PV module voltage and load range. IEEE Transactions on Industrial Electronics 2008; 55(7):2655-2663.

(10) Su G-J, Tang L. A multiphase, modular, bidirectional, triple-voltage DC-DC converter for hybrid and fuel cell vehicle power systems. IEEE Transactions on Power Electronics 2008; 23(6):3035-3046.

(11) Sangtaek H, Divan D. Bi-directional DC/DC converters for plugin hybrid electric vehicle (PHEV) applications. APEC 2008; 1: 784-789.

(12) Chiu H-J, Lin L-W. A bidirectional DC-DC converter for fuel cell electric vehicle driving system. IEEE Transactions on Power Electronics 2006; 21(4):950-958.

(13) Inoue S, Akagi H. A bi-directional isolated DC/DC converter as a core circuit of the next-generation medium-voltage power conversion system. IEEE Transactions on Power Electronics 2007; 22(2):535-542.

(14) Wu X, Xie X, Zhao C, Qian Z, Zhao R. Low voltage and current stress ZVZCS full bridge DC-DC converter using center tapped rectifier reset. IEEE Transactions on Industrial Electronics 2008; 55(3):1470-1477.

(15) Xiao H, Xie S. A ZVS bidirectional DC-DC converter with phaseshift plus PWM control scheme. IEEE Transactions on Power Electronics 2008; 23(2):813-823.

(16) Sosa JL, Castilla M, Miret J, Garcia de Vicuna L, Matas J. Modeling and performance analysis of the DC/DC series - parallel resonant converter operating with discrete self-sustained phase-shift modulation technique. IEEE Transactions on Industrial Electronics 2009; 56(3):697-705.

(17) Oliveira AC, Jacobina CB, Lima AMN. Improved dead-time compensation for sinusoidal PWM inverters operating at high switching frequencies. IEEE Transactions on Industrial Electronics 2007; 54(4):2295-2304.

(18) Bai H, Mi C, Gargies S. The short-timescale transient processes in high-voltage and high-power isolated bidirectional DC-DC converters. IEEE Transactions on Power Electronics 2008; 23(6):2648-2656.

(19) Bai H, Zhao Z, Mi C. Framework and research methodology of short-timescale pulsed power phenomena in high voltage and high power converters. IEEE Transactions on Industrial Electronics 2009; 56(3):805-816.

(20) Bai H, Mi C, Wang C, Gargies S. The dynamic model and hybrid phase-shift control of a bidirectional dual active bridge DC-DC converter. IECON 2008; 1: 2840-2845.

(21) Mi C, Bai H, Wang C, Gargies S. The operation, design, and control of dual H-bridge based isolated bidirectional DC-DC converter. IET Power Electronics 2008; 1(4):507-517.

(22) Bai H, Mi C. Eliminate reactive power and increase system efficiency of isolated bidirectional dual-active-bridge DC-DC converters using novel dual-phase-shift control. IEEE Transactions on Power Electronics 2008; 23(6):2905-2914.

Hua Bai (Non-member) was born in Chifeng City, Inner Mon-

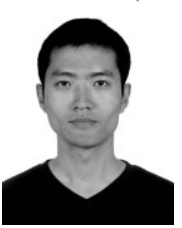
golia, China in 1980. He received the B.S. and $\mathrm{Ph} . \mathrm{D}$. degrees in the Department of Electrical Engineering of Tsinghua University, Beijing, China in 2002 and 2007, respectively. He was a post-doc fellow and research scientist in University of Michigan-Dearborn, USA, in 2007 and 2009, respectively. Now he is an Assistant Professor in the Department of Electrical and Compurter Engineering, Kettering University, MI, USA. His research interest is the dynamic processes and transient pulsed power phenomena of power electronic devices, including variable frequency motor drive system, high voltage and high power DC/DC converter, renewable energy, and hybrid electric vehicles. 
Ziling Nie (Non-member) received the B.S. (with honors),

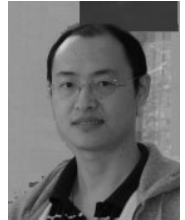

M.S., and Ph.D. degrees from Huazhong University of Science and Technology (HUST), China, in 1998, 2001, and 2005, respectively. At Huazhong University of Science and Technology (2006-2008), he was a Research Assistant at the Power Electronics Research Laboratory and a Teaching Assistant in the EECS Department. In 2008, he joined the University of Michigan-Dearborn Laboratory for Power Electronics as a Postdoctoral Associate. His research interests include power electronics, automotive electrical/electronic systems, and computer-aided modeling/simulation.
Chris Mi (Non-member) received the B.S. and M.S. degrees from

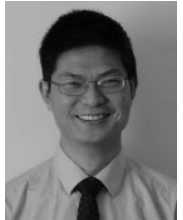
Northwestern Polytechnical University, China, and the Ph.D. degree from the University of Toronto, Canada, all in electrical engineering. $\mathrm{He}$ is associate professor at the University of Michigan-Dearborn and the vice chair of the IEEE Southeast Michigan section. Dr Mi worked with General Electric Company from 2000 to 2001. He won the 'National Innovation Award', the 'Government Special Allowance', and winner of the 2005 'Distinguished Teaching Award' of University of Michigan-Dearborn. He is a recipient of the 2007 IEEE Region 4 'Outstanding Engineer Award', and the 2007 IEEE Southeastern Michigan Section 'Outstanding Professional Award'. He recently won the 2007 SAE E2T Award. 